\title{
Survival, Motor Function, and Motor Milestones: Comparison of AVXS-101 Relative to Nusinersen for the Treatment of Infants with Spinal Muscular Atrophy Type 1
}

\author{
Omar Dabbous - Benit Maru · Jeroen P. Jansen • Maria Lorenzi • \\ Martin Cloutier · Annie Guérin · Irina Pivneva • Eric Q. Wu • \\ Ramesh Arjunji · Douglas Feltner · Douglas M. Sproule
}

Received: December 14, 2018 / Published online: March 16, 2019

(C) The Author(s) 2019

\section{ABSTRACT}

Introduction: Infants with spinal muscular atrophy (SMA) type 1 typically face a decline in motor function and a severely shortened life expectancy. Clinical trials for SMA type 1 therapies, onasemnogene abeparvovec (AVXS-101) and nusinersen, demonstrated meaningful improvements in efficacy (e.g., overall survival) but there were no head-to-head clinical trials assessing comparative efficacy. This study estimated the treatment effects of AVXS-101 relative to nusinersen for the treatment of SMA type 1 .

Methods: Overall survival, event-free survival (no death or need to use permanent assisted ventilation), improvement in motor function [increase of $\geq 4$ points in Children's Hospital of

Enhanced Digital Features To view enhanced digital features for this article go to https://doi.org/10.6084/ m9.figshare.7775933.

O. Dabbous · B. Maru · R. Arjunji · D. Feltner .

D. M. Sproule $(\bowtie)$

AveXis, Inc., Bannockburn, IL, USA

e-mail: dsproule216@avexis.com

J. P. Jansen · M. Lorenzi

Precision Xtract, Oakland, CA, USA

M. Cloutier · A. Guérin · I. Pivneva

Analysis Group, Inc., Montreal, Canada

E. Q. $\mathrm{Wu}$

Analysis Group, Inc., Boston, MA, USA
Philadelphia Infant Test of Neuromuscular Disorders (CHOP-INTEND) score from baseline], and motor milestone achievements (head control, rolling over, and sitting unassisted) reported in the onasemnogene abeparvovec (AVXS101-CL-101; NCT02122952) and nusinersen (ENDEAR; NCT02193074) clinical trials were indirectly compared using frequentist and Bayesian approaches.

Results: Among symptomatic infants with SMA type 1 , the number needed to treat (NNT) to prevent one more death with AVXS-101 instead of nusinersen was 6.2 [95\% confidence intervals $(\mathrm{CI})=4.1-12.2]$, and the probability of preventing death was $20 \%$ higher for patients treated with AVXS-101 than nusinersen [risk ratio $(\mathrm{RR})=1.2,95 \% \mathrm{CI} 1.1-1.3]$. For event-free survival, the NNT to prevent one more event was 2.6 (95\% CI 2.0-3.6) and RR was 1.6 (95\% CI 1.4-1.9). For improvement in motor function, NNT was 3.5 (95\% CI 2.6-5.3) and RR was 1.4 (95\% CI 1.2-1.6). For milestone achievements, the NNTs were 1.4 (95\% CI 1.1-1.9), 1.5 (95\% CI 1.1-2.5), and 1.2 (95\% CI 1.0-1.5); RRs 4.2 (95\% CI 2.6-6.7), 7.8 (95\% CI 3.6-17.0), and 11.2 (95\% CI 5.1-24.5) for head control, rolling over, and sitting unassisted, respectively. Results were similar using the Bayesian approach.

Conclusion: This indirect comparison (AVXS101-CL-101 vs. ENDEAR) among symptomatic SMA type 1 infants suggests that AVXS-101 may have an efficacy advantage relative to 
nusinersen for overall survival, independence from permanent assisted ventilation, motor function, and motor milestones.

Funding: AveXis.

Keywords: AVXS-101; Indirect treatment comparison; Nusinersen; Neuroscience; Onasemnogene abeparvovec; Spinal muscular atrophy type 1 (SMA type 1)

\section{INTRODUCTION}

Spinal muscular atrophy (SMA) is a progressive and debilitating severe neurodegenerative disease with an incidence of approximately 1 in 10,000 live births [1-4]. It is a monogenic disorder characterized by mutations in the survival motor neuron 1 (SMN1) gene and insufficient production of functional SMN protein causing degeneration and loss of motor neurons resulting in muscle weakness and atrophy and paralysis, as SMN protein is ubiquitously expressed and plays a role in muscle functioning [3, 5-7].

SMA is clinically classified into four subtypes (SMA types 1-4) based on the severity of symptoms and the age of onset [1, 3, 8, 9]. The number of copies of the survival motor neuron 2 (SMN2) gene, a modifier gene nearly identical to SMN1 that produces a small, insufficient fraction of SMN protein, is a major determinant of this variability in the clinical phenotype [10]. The most common and most severe form of SMA is type 1 (SMA type 1), also known as infantile onset, accounting for approximately $60 \%$ of cases $[1,8,11,12]$. Infants with SMA type 1 most often become symptomatic within the 1st months of life by failing to reach basic developmental motor milestones, such as the ability to sit without support, and often face a decline in motor function as assessed by standardized methods, such as the Children's Hospital of Philadelphia Infant Test of Neuromuscular Disorders (CHOP-INTEND) [12-14].

Rapidly progressive muscle weakness in SMA type 1 can impede bodily functions, particularly respiratory and swallowing functions, further requiring mechanical nutritional and respiratory (e.g., permanent assisted ventilation) support. Even with proactive nutritional and respiratory support, infants with SMA type 1 typically face a decline in motor function and have a severely shortened life expectancy, rarely surviving beyond infancy $[1,8,13]$. An observational study of SMA type 1 by Finkel and colleagues [13] reported that infants with SMA type 1 (and 2 copies of SMN2) had a median survival of 10.5 months without death or respiratory support (defined as $\geq 16$ hours per day of non-invasive ventilatory support or tracheostomy) with only $8 \%$ surviving to 20 months free of such respiratory support (see also [15-17]).

There has been substantial progress in the development of effective therapies for SMA $[3,8]$. Nusinersen, an antisense oligonucleotide drug designed to enhance the production of SMN protein by modifying pre-messenger RNA splicing of $S M N 2$, was the first drug to be approved by the Food and Drug Administration (FDA) to treat SMA, in December 2016 [18]. Nusinersen has demonstrated significant efficacy in improving event-free survival, motor function, and motor milestones in infants with SMA type 1 [19]. However, given that nusinersen does not cross the blood-brain barrier when delivered systemically and has a median half-life of 163 days, it requires direct delivery into the central nervous system through lifetime repeated intrathecal injections [20].

Recently, a phase 1, open-label, single-infusion, ascending dose, single-center clinical trial assessing gene-replacement therapy with onasemnogene abeparvovec (AVXS-101) in SMA type 1 infants was completed (AVXS-101CL-101; NCT02122952). AVXS-101 uses a nonreplicating adeno-associated virus capsid to deliver a functional copy of the $S M N$ gene to motor neuron cells in SMA patients as a onetime intravenous injection. A key difference of AVXS-101 is that it can cross the blood-brain barrier allowing for effective central nervous system delivery in a single intravenous injection of the drug to target motor neurons in the central nervous system [21].

Despite some differences in clinical trial design and study outcomes, clinical trials of AVXS-101 and nusinersen in symptomatic infants with SMA type 1 both assessed eventfree survival (i.e., time until death or the need 
for permanent ventilator assistance), motor function using CHOP-INTEND scores, and motor milestone achievements. Although clinical trials of AVXS-101 and nusinersen both demonstrated efficacy of the respective drugs in improving event-free survival, motor function, and motor milestone achievements, there is limited information on the comparative efficacy of the two treatments as there have been no head-to-head clinical trials. In the absence of a head-to-head trial, a between-trial comparison of the reported results of the two trials is currently the only method of comparison of relative treatment effects of nusinersen and AVXS101.

The objective of this study was to compare AVXS-101 with nusinersen for the treatment of patients with SMA type 1 in terms of overall survival, the need to use permanent assisted ventilation, improvement in motor function, and motor milestone achievement based upon the results of two clinical trials in symptomatic infants with SMA type 1.

\section{METHODS}

\section{Evidence Base}

At the time of the present study, AVXS-101 and nusinersen (FDA-approved treatment) were the only therapies with recently published data from clinical trials suggesting efficacy for the treatment of SMA type 1 in infants. The most recently published results of clinical trials of AVXS-101 (AVXS-101-CL-101; NCT02122952) [21] and nusinersen (ENDEAR; NCT02193074) [19] were used for the present study. AVXS-101 phase I was the only AVXS-101 clinical trial with published findings. ENDEAR, a phase III, double-blind, randomized study of nusinersen and placebo was the most recently published nusinersen clinical trial comprised of a larger sample than previously published clinical trials with similar outcome measures to the AVXS-101 phase I clinical trial. Two cohorts were enrolled in the AVXS-101 clinical trial: patients in cohort $1(n=3)$ received a minimally effective dose $\left(6.7 \times 10^{13} \mathrm{vg}\right.$ per kilogram) and patients in cohort $2(n=12)$ received the proposed therapeutic dose $\left(2.0 \times 10^{14} \mathrm{vg}\right.$ per kilogram) [21]. Of note, the proposed therapeutic dose at the time the clinical trial was conducted was based on unvalidated quantitative polymerase chain reaction (qPCR). The current proposed therapeutic dose for AVXS101 is $1.1 \times 10^{14}$ vg per kilogram based on validated droplet digital polymerase chain reaction (ddPCR). The current comparative study focuses on outcomes for patients in cohort 2. Key clinical trial characteristics are described in Table 1 , and more detailed descriptions of both clinical trials are available in previous publications [19, 21].

\section{Outcomes}

The outcomes considered in the current study were those that were assessed in both clinical trials (i.e., similar outcomes in both clinical trials), namely survival, the need to use permanent assisted ventilation, event-free survival, motor function, and motor milestone achievements (Table 1). Event-free survival was defined as no death and no need to use permanent assisted ventilation and was evaluated at last visit. In addition, no death and no need to use permanent assisted ventilation were analyzed separately. In AVXS-101-CL-101, permanent assisted ventilation was defined as $\geq 16 \mathrm{~h}$ of respiratory assistance per day continuously for $\geq 14$ days in the absence of an acute, reversible illness or a perioperative state, while in ENDEAR, it was defined as tracheostomy or ventilatory support for $\geq 16 \mathrm{~h}$ per day continuously for $>21$ days in the absence of an acute reversible event. Motor function treatment effect was assessed by a CHOP-INTEND response, defined as an increase of $\geq 4$ points from baseline, and was recorded at the last study visit. For the nusinersen clinical trial, the CHOP-INTEND response was only reported among patients who were enrolled for at least 6 months. For the AVXS-101 clinical trial, the CHOP-INTEND response was adapted from the definition in the nusinersen clinical trial and was calculated from the patient-level data. Motor milestone achievements were defined as a milestone that would be expected for normal motor development at a particular age in infancy and included head control, rolling over, and sitting unassisted [22]. 


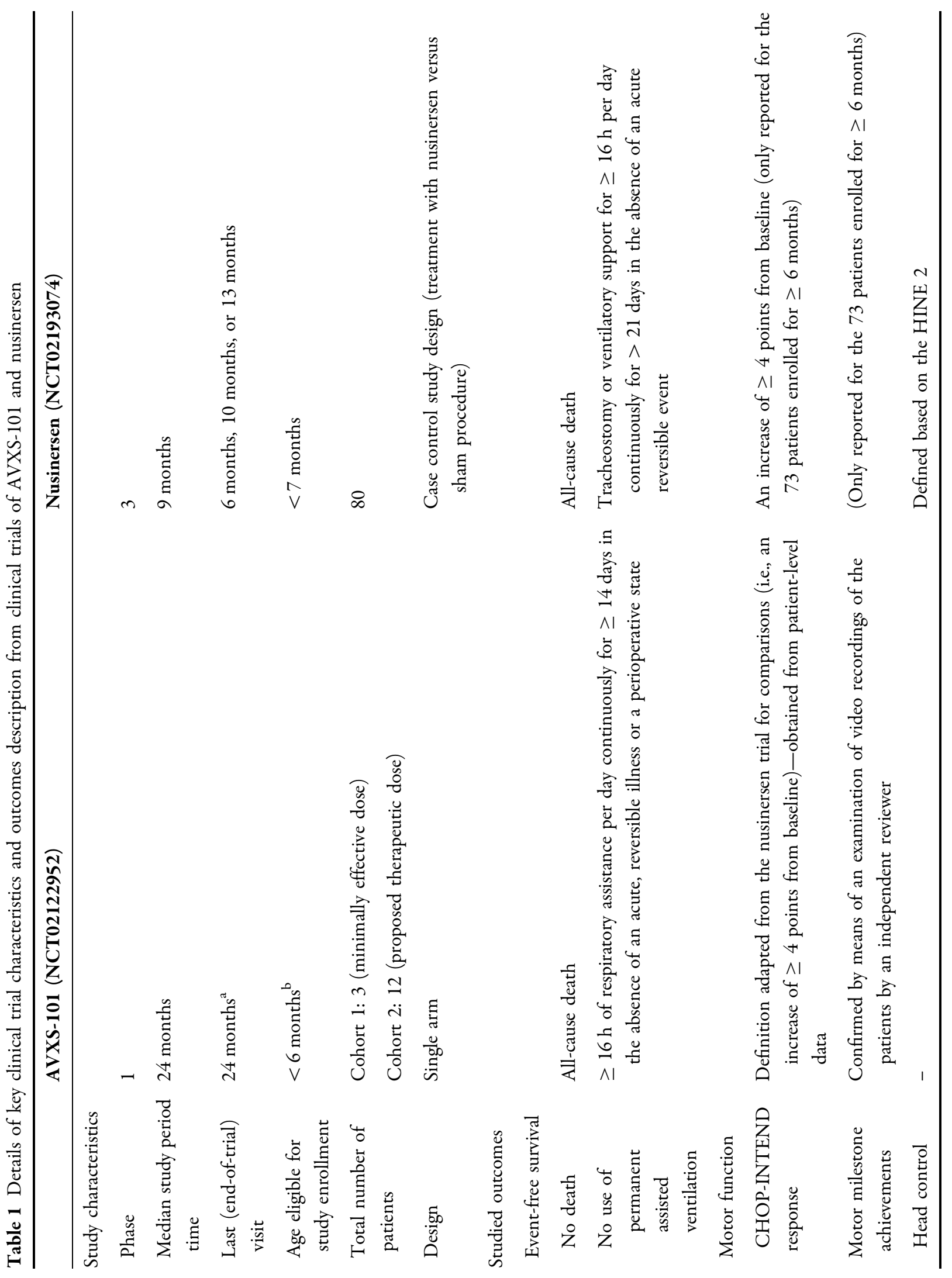




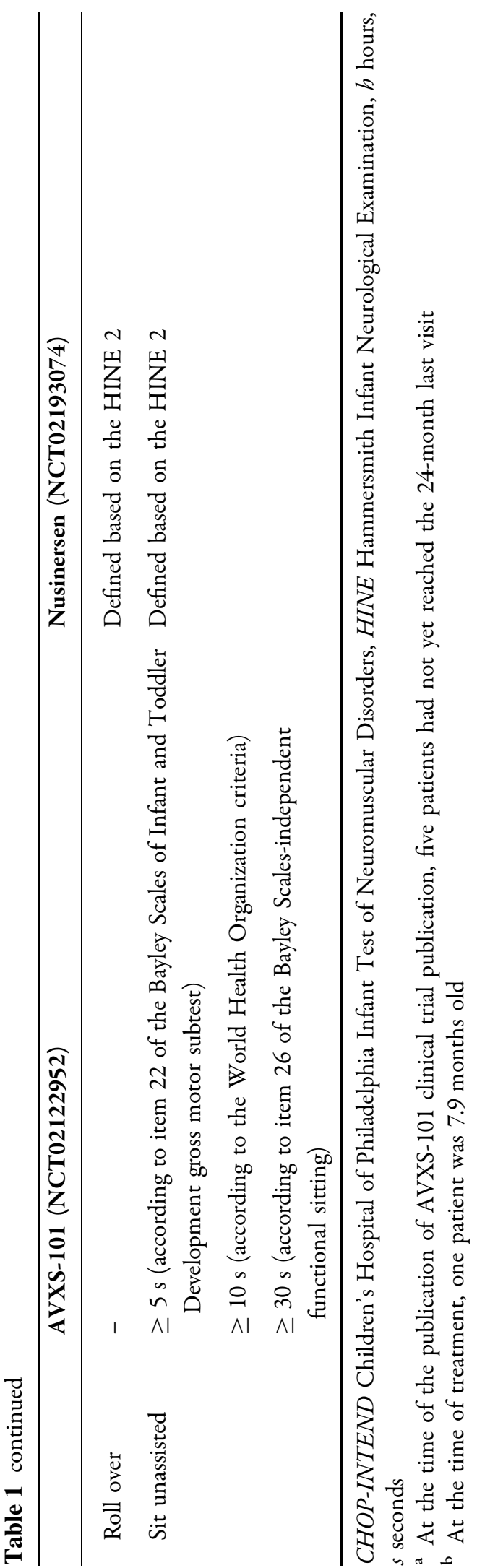

The outcomes were assessed at the last visit as defined by their respective clinical trials. For the AVXS-101 clinical trial, the last visit occurred at 24 months, though at the time of the publication of the AVXS-101 clinical trial, five patients had not yet reached the 24-month last visit. For the nusinersen clinical trial, the last visit occurred at 6,10 , or 13 months. Because AVXS-101 and nusinersen clinical trials had a different trial duration, the CHOP-INTEND response was also evaluated at 9 months (median time on trial for nusinersen). CHOPINTEND scores at 9 months were imputed for three patients with missing data in AVXS-101 (cohort 2) clinical trial; because each of the three patients consistently had an increase of $\geq 4$ points both during the visit before and after month 9 , these patients were assumed to have had an increase of $\geq 4$ points at 9 months.

\section{Statistical Analyses}

Two approaches, frequentist and Bayesian, were used to compare the treatment effects regarding outcomes of interest from the AVXS-101 and nusinersen clinical trials. NNT was obtained for patients treated with AVXS-101 (cohort 2) from the AVXS-101 clinical trial versus patients treated with nusinersen from the nusinersen clinical trial. The NNT to prevent one additional death with AVXS-101 relative to nusinersen was calculated as the reciprocal of the difference between AVXS-101 and nusinersen in the estimated probability of survival. The NNTs to prevent the need to use permanent assisted ventilation, one event (death or the need to use permanent assisted ventilation), to achieve one additional improved motor function, and to achieve a motor milestone with AVXS-101 relative to nusinersen were calculated in a similar manner. The NNT was evaluated for each outcome and was reported with 95\% confidence intervals (CIs). Lower NNT denotes higher treatment efficacy (i.e., fewer patients need to receive the treatment to see a benefit). In addition, risk differences (RD) and relative risks (RR) with their corresponding 95\% CIs were calculated for patients treated with AVXS-101 versus nusinersen for each of the selected outcomes. 
Table 2 Patient characteristics among patients from the AVXS-101 $(N=12)$ and nusinersen $(N=80)$ clinical trials

\begin{tabular}{|c|c|c|}
\hline Patient characteristics & $\begin{array}{l}\text { AVXS-101 } \\
N=12\end{array}$ & $\begin{array}{l}\text { Nusinersen } \\
N=\mathbf{8 0}\end{array}$ \\
\hline Female sex, $N(\%)$ & $7(58.3 \%)$ & $43(53.8 \%)$ \\
\hline \multicolumn{3}{|l|}{ Race, $N(\%)$} \\
\hline White & $11(91.7 \%)$ & - \\
\hline Other & $1(8.3 \%)$ & - \\
\hline \multicolumn{3}{|l|}{ Symptoms of SMA, $N(\%)$} \\
\hline Hypotonia & $12(100.0 \%)$ & $80(100.0 \%)$ \\
\hline Limb weakness & $11(91.7 \%)$ & $79(98.8 \%)$ \\
\hline Swallowing or feeding difficulties & $5(41.7 \%)$ & $41(51.3 \%)$ \\
\hline Other & 0 & $20(25.0 \%)$ \\
\hline Mean age at symptom onset, months (range) & $1.4(0-3.0)$ & $1.8(0.5-4.1)$ \\
\hline Mean age at genetic diagnosis, days (range) & $60.0(0-136.0)$ & $88.2(0-203.0)$ \\
\hline Mean age at first dose, months (range) & $3.4(0.9-7.9)$ & $5.3(1.7-7.9)$ \\
\hline Mean weight at first dose, $\mathrm{kg}$ (range) & $5.7(3.6-8.4)$ & - \\
\hline Disease duration at screening, weeks, mean (range) & - & $13.2(0-25.9)$ \\
\hline \multicolumn{3}{|l|}{ Patients with clinical support at baseline, $N(\%)$} \\
\hline Nutritional $^{\mathrm{b}}$ & $5(41.7 \%)$ & $7(8.8 \%)$ \\
\hline Ventilatory ${ }^{c}$ & $2(16.7 \%)$ & $21(26.3 \%)$ \\
\hline Mean CHOP-INTEND score at baseline ${ }^{\mathrm{d}}$ & 28.2 & 26.6 \\
\hline Mean HINE-2 score at baseline ${ }^{\mathrm{e}}$ & - & 1.29 \\
\hline
\end{tabular}

"-" Indicates that the variable was possibly collected but not reported in the corresponding publication CHOP-INTEND Children's Hospital of Philadelphia Infant Test of Neuromuscular Disorders, HINE Hammersmith Infant Neurological Examination, $\mathrm{kg}$ kilogram, $N$ number, $S M A$ spinal muscular atrophy

${ }^{a}$ Some descriptive statistics reported in the table from the nusinersen clinical trial were converted from months or weeks into days to match those reported by the AVXS-101 clinical trial

b Nutritional support included gastrostomy or nasogastric tube for AVXS-101 and gastrointestinal tube for nusinersen

${ }^{c}$ Ventilatory support at baseline was not defined in studies for either AVXS-101 or nusinersen

d Scores on the CHOP-INTEND range from 0 to 64, with higher scores indicating better motor function

e Scores on HINE-2 range from 0 to 26, with higher scores indicating better motor function

Similar to NNT calculations, RD and RRs were based on AVXS-101 cohort 2.

The Bayesian framework does not require an assumption that the effect estimates be normally distributed in calculating the standard errors and CIs, and it further allows using the exact likelihood specifications and synthesizing sparse data without any modifications. The Bayesian framework was based on non- informative prior distributions for the parameters representing the relative treatment effects between AVXS-101 and nusinersen.

\section{Compliance with Ethics Guidelines}

This article is based on previously conducted studies and does not contain any studies with 


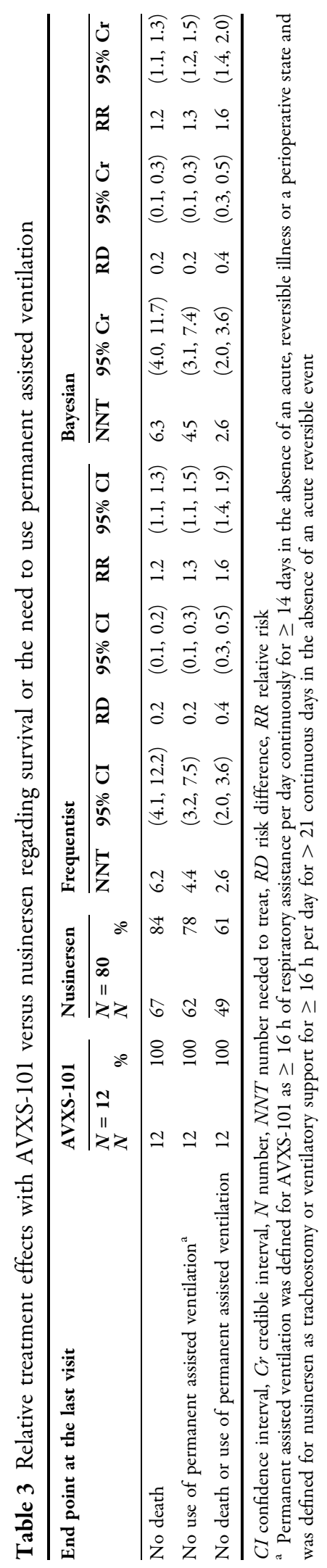

human participants or animals performed by any of the authors.

\section{RESULTS}

Characteristics of patients treated with AVXS101 and nusinersen from their respective clinical trials are summarized in Table 2. Reported mean age (range) at symptom onset was 1.4 (0-3.0) months for patients in the AVXS-101 clinical trial and $1.8(0.5-4.1)$ months for patients in the nusinersen clinical trial. Patient mean age (range) at first dose was 3.4 (0.9-7.9) and 5.3 (1.7-7.9) months for patients in the AVXS-101 and nusinersen clinical trials, respectively; $58.3 \%$ of patients in the AVXS-101 clinical trial and $53.8 \%$ of patients in the nusinersen clinical trial were female. The mean CHOP-INTEND score at baseline was 28.2 and 26.6 for patients in the AVXS-101 and nusinersen clinical trials, respectively.

In terms of survival, $100 \%$ of patients in the AVXS-101 clinical trial were alive at the last visit, whereas $84 \%$ of patients in the nusinersen clinical trial were alive at the last visit (Table 3 ). The NNT to prevent one more death with AVXS-101 instead of nusinersen was estimated at 6.2 , and the probability of preventing death was $20 \%$ higher for patients treated with AVXS101 than for those treated with nusinersen $(\mathrm{RR}=1.2)$. In terms of event-free survival, no death and no need to use permanent assisted ventilation were reported in $100 \%$ of patients in the AVXS-101 clinical trial and $61 \%$ for patients in the nusinersen clinical trial. The NNT to prevent one more event with AVXS-101 instead of nusinersen was estimated at 2.6, and RR was estimated at 1.6. Similar results were obtained using the Bayesian approach (Table 3).

In terms of motor function, all patients in the AVXS-101 clinical trial achieved a CHOPINTEND response (i.e., an increase of $\geq 4$ points from baseline), whereas the proportion was $71 \%$ for patients in the nusinersen clinical trial (both at last visit and over a median of 9 months; Table 4). The NNT to have one more patient improve motor function (i.e., an increase in CHOP-INTEND score of $\geq 4$ points from the baseline) with AVXS-101 instead of nusinersen 


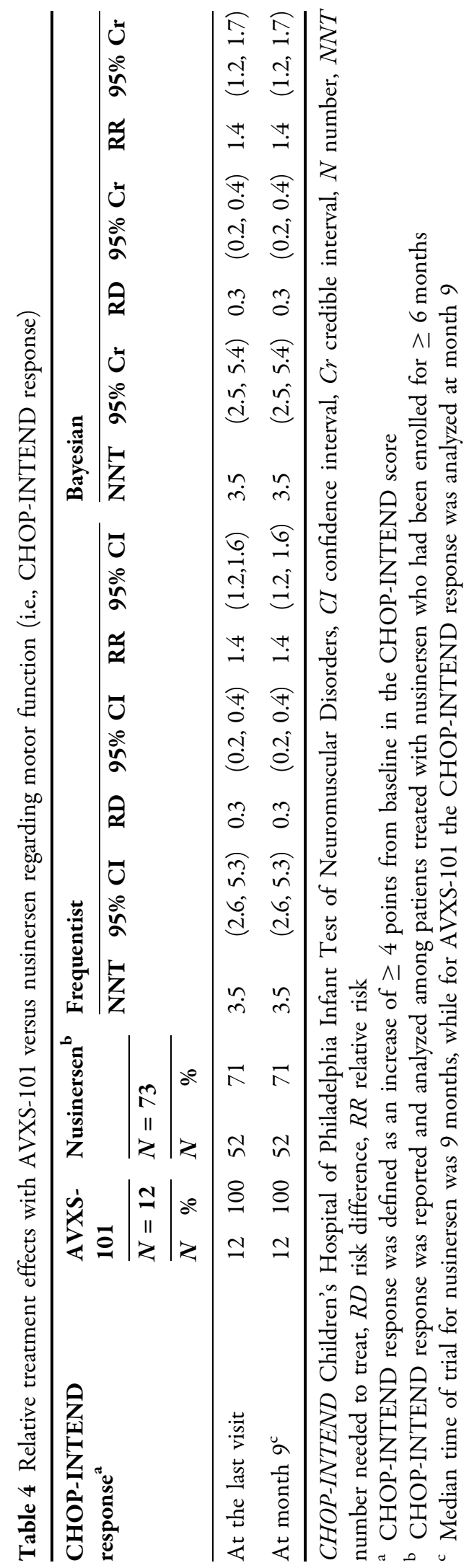

was estimated at 3.5. The probability of attaining a CHOP-INTEND response (i.e., increase of $\geq 4$ points from baseline) was $40 \%$ higher for patients treated with AVXS-101 than for those treated with nusinersen (both at last visit and over a median of 9 months; both RRs $=1.4$ ). Similar results were obtained using a Bayesian approach (Table 4).

In terms of motor milestone achievements, $92 \%$ of patients in the AVXS-101 clinical trial achieved head control at the last visit, whereas the proportion was $22 \%$ for patients in the nusinersen trial (Table 5). The NNT to have one more patient achieve head control with AVXS101 instead of nusinersen was estimated at 1.4. The probability of achieving head control was 4.2 times higher for patients treated with AVXS101 than for patients treated with nusinersen. In terms of rolling over, $75 \%$ of patients in the AVXS-101 clinical trial could roll over at the last visit, whereas the proportion was $10 \%$ for patients in the nusinersen clinical trial. The NNT to have one more patient roll over with AVXS-101 instead of nusinersen was estimated at 1.5, and RR was estimated at 7.8. In terms of sitting, $92 \%$ of patients in the AVXS-101 clinical trial could sit unassisted for $\geq 5 \mathrm{~s}$ at the last visit and $75 \%$ for $\geq 30 \mathrm{~s}$, whereas $8 \%$ of patients could sit unassisted in the nusinersen clinical trial. The NNT to have one more patient sit unassisted (for $\geq 5 \mathrm{~s}$ ) with AVXS-101 instead of nusinersen was estimated at 1.2 and RR was estimated at 11.2. Similar results were observed using a Bayesian approach (Table 5).

\section{DISCUSSION}

This study compared the efficacy of two novel therapies for SMA type 1, AVXS-101 and nusinersen, based upon a comparison of the efficacy results reported in their respective clinical trials of symptomatic infants with SMA type 1 . Both therapies have demonstrated significant improvements in survival, motor function, and motor milestones in patients treated in their respective clinical trials, and the indirect comparison in the current study potentially suggests that AVXS-101 may be associated with greater clinical benefits than 


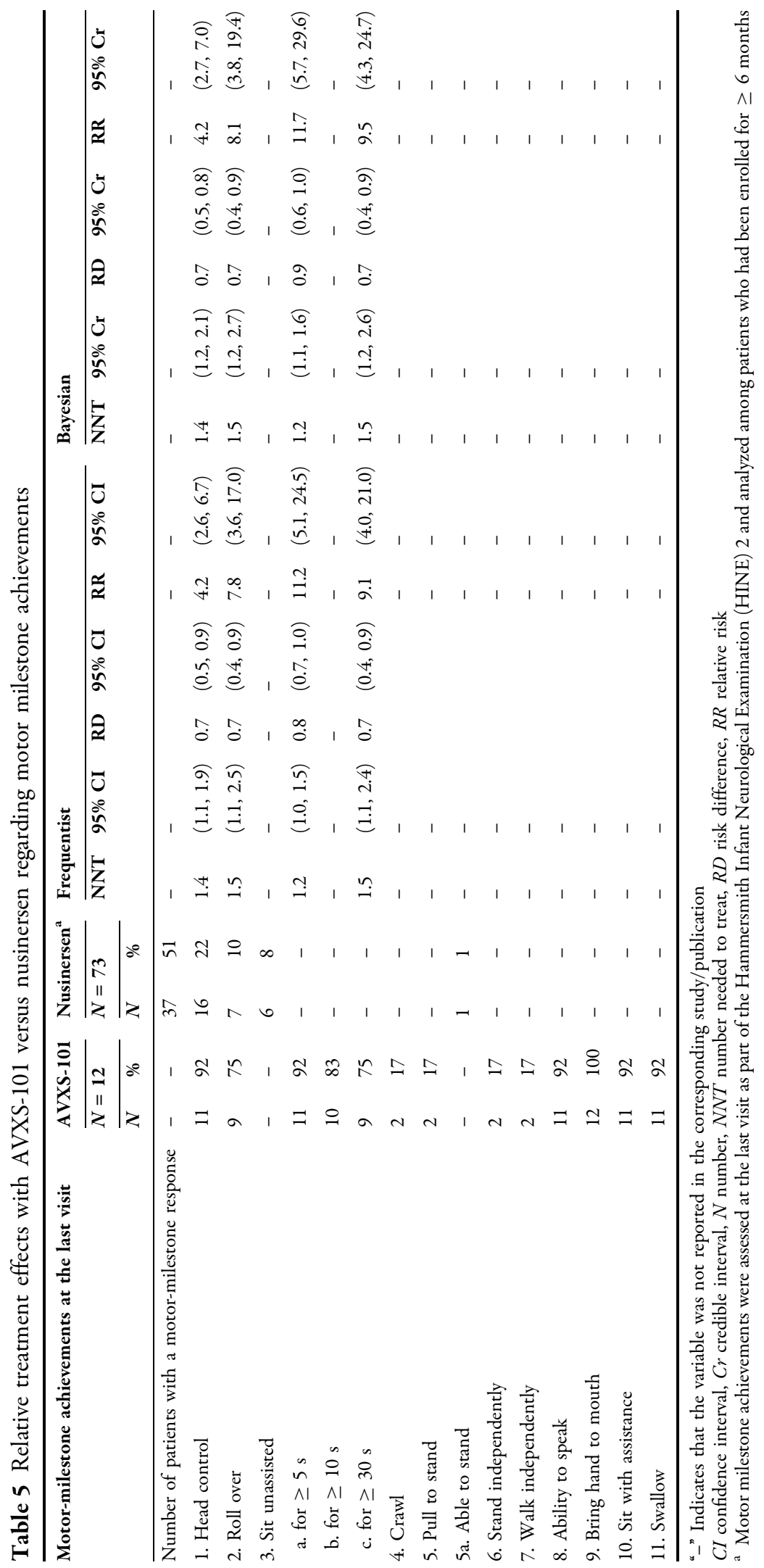


what was observed for nusinersen [19, 21]. Compared with nusinersen, AVXS-101 appears to have favorable efficacy outcomes across survival, motor function, and motor milestones. For each 2.6 patients treated with AVXS-101 instead of nusinersen, one more death or the need to use permanent assisted ventilation can be prevented, and the probability of preventing death or the need to use permanent assisted ventilation was $60 \%$ higher for AVXS-101 compared with nusinersen. AVXS-101 was also more favorable compared with nusinersen with respect to overall survival, motor function as indexed by attaining a CHOP-INTEND response, and motor milestone achievements. The results were very similar when analyses were conducted within the Bayesian framework.

This study did not compare adverse event rates associated with the two interventions of interest. Although both clinical trials provided information on adverse events, the type of and the manner in which the adverse events were reported across clinical trials were different, likely because of different mechanisms of action, hence hindering comparisons. In addition, several events reported are likely diseaserelated rather than treatment-related. Adverse event rates for each treatment are reported in their respective clinical trial $[19,21]$.

In addition to the improved efficacy across survival, motor function, and motor milestones observed in this study versus nusinersen in ENDEAR, an important difference of AVXS-101 is that it requires a single intravenous infusion, with no requirements for substantial specialized resources and medical expertise, thus overcoming challenges with administration, access, and adherence [23]. Altogether, the advantages of AVXS-101 across survival, motor function, and motor milestones suggest that AVXS-101 may contribute to improving patients' and caregivers' quality of life, particularly assuming that a onetime treatment with AVXS-101 might translate into increased survival and the ability to improve motor function and motor milestone achievement over time. Similarly, AVXS-101 might potentially provide additional benefits in the longer term regarding reduced medical resource use (e.g., fewer visits to the hospital, reduced need for ambulatory care) and relating to reduced medical costs.
This study should be interpreted in the context of some limitations. First, this study was based on published results from clinical trials of AVXS-101 and nusinersen; thus, it was not a head-to-head study. In the absence of a control group for AVXS-101, as it was a single-arm openlabel trial, the indirect comparison was unanchored (i.e., indirect comparison through a common comparator arm), assuming similar distributions of prognostic variables and effects modifiers between clinical trials; the comparison was conducted based on the best evidence available. To that effect, the single-arm, historicalcontrolled trial study design for the AVXS-101 clinical trial might still be deemed appropriate because of ethical concerns with a randomized controlled trial for a condition that does not naturally remit and carries very little survival beyond infancy for historic controls [12]. In addition, the unavailability of individual patientlevel data for the nusinersen clinical trial did not allow for direct comparisons (e.g., case-matched analyses) or other forms of indirect comparisons, including adjustments for potential confounding factors. Although the two clinical trials were similar with respect to some patient characteristics at the time of enrollment (e.g., the CHOPINTEND scores were 28 and 26.6 points among patients in the AVXS-101 and nusinersen clinical trials, respectively), the results reported should be interpreted in light of potentially important differences in both measured and unmeasured characteristics between the clinical trial populations and also differences in trial design. Notably, the AVXS-101 and nusinersen clinical trials were different with respect to mean age at first dose (3.4 vs. 5.3 months) and dependence on ventilatory support at baseline (17\% vs. $26 \%)$, among others $[19,21]$. To that extent, more favorable outcomes can be achieved if patients start treatment earlier; thus, the difference in mean age at which patients initiated treatment across cohorts might affect the results of the study as patients were not matched for age or disease burden. In addition, some differences in study outcomes with respect to time points (e.g., differences in median duration of follow-up) and patient characteristic definitions (e.g., need for nutritional support at enrollment) were present. The longer follow-up period in the AVXS-101 clinical trial 
compared with the nusinersen clinical trial could create a bias against AVXS-101 for mortality outcomes while resulting in a possible advantage for motor milestone outcomes by allowing more time for patients to achieve these milestones. Nevertheless, despite differences in time points at which study outcomes were reported, given that AVXS-101 survival-related outcomes were reported at a later time (i.e., 24 months) than those for nusinersen, all patients were alive and would still be alive if assessed at earlier time points. In addition, these patients would be alive and exceed the survival observed in the natural history of the disease where few infants will survive beyond 2 years of age without significant ventilatory support $[12,13,17,24-26]$. Finally, both clinical trials had relatively short follow-up durations. As long-term effects of both treatments are currently unknown, long-term monitoring to assess the duration of benefit and sequelae of both therapies over a longer time period is essential. The findings of this study should be interpreted in the context of these limitations; nevertheless, the results are based on the best evidence to date regarding comparative efficacy between AVXS-101 and nusinersen treatments.

\section{CONCLUSIONS}

Based upon a comparison of the AVXS-101-CL101 and ENDEAR clinical trials in symptomatic infants with SMA type 1, the findings of this study suggest AVXS-101 may have an efficacy advantage relative to nusinersen in terms of overall survival, the need to use permanent assisted ventilation, motor function, and motor milestones. Long-term monitoring of patients treated with AVXS-101 is needed to confirm maintenance of observed effects over longer time periods.

\section{ACKNOWLEDGEMENTS}

Funding. Sponsorship for this study, article processing charges, and open access fees were funded by AveXis. All authors had full access to all of the data in this study and take complete responsibility for the integrity of the data and accuracy of the data analysis.

Medical Writing, Editorial, and other Assistance. Medical writing assistance in the preparation of this article was provided by Dr. Sara Kaffashian, an employee of Analysis Group, Inc.; support for this assistance was provided by AveXis.

Authorship. All named authors meet the International Committee of Medical Journal Editors (ICMJE) criteria for authorship for this article, take responsibility for the integrity of the work as a whole, and have given their approval for this version to be published.

Previous Presentations. Preliminary data of frequentist approach were presented at the International Society for Pharmacoeconomics and Outcomes Research 23rd Annual International Meeting, 19-23 May 2018, Baltimore, MD, USA; at the International Congress on Neuromuscular Diseases, 6-10 July 2018, Vienna, Austria; at the World Muscle Society, 2-6 October 2018, Mendoza, Argentina; Academy of Managed Care Pharmacy Nexus, 22-25 October 2018, Orlando, FL, USA; and have been accepted for presentation at the Academy of Managed Care Pharmacy, on 25-28 March 2019, San Diego, CA, USA. Preliminary data of Bayesian approach were presented at the International Society for Pharmacoeconomics and Outcomes Research Europe, 10-14 November 2018, Barcelona, Spain, and at the European Society for Cell and Gene Therapy, Lausanne, Switzerland, 16-19 October 2018.

Disclosures. Benit Maru is an employee of SSI Strategy, who are contracted to support AveXis, Inc. Jeroen P. Jansen is an employee of Precision Xtract, which received consultancy fees from AveXis Inc. for this work. Maria Lorenzi is an employee of Precision Xtract, which received consultancy fees from AveXis Inc. for this work. Omar Dabbous is an employee of AveXis. Douglas Feltner is an employee of AveXis. Douglas M. Sproule is an employee of AveXis. Martin Cloutier is an employee of Analysis Group, Inc., which has received 
consultancy fees from AveXis for this work. Annie Guérin is an employee of Analysis Group, Inc., which has received consultancy fees from AveXis for this work. Irina Pivneva is an employee of Analysis Group, Inc., which has received consultancy fees from AveXis for this work. Eric Q. Wu is an employee of Analysis Group, Inc., which has received consultancy fees from AveXis for this work. Ramesh Arjunji is an employee of Avexis, Inc., a Novartis Co., and may own AveXis/Novartis stock or other equities.

Compliance with Ethics Guidelines. This article is based on previously conducted studies and does not contain any studies with human participants or animals performed by any of the authors.

Data Availability. Data sharing is not applicable to this article as no data sets were generated or analyzed during the current study.

Open Access. This article is distributed under the terms of the Creative Commons Attribution-NonCommercial 4.0 International License (http://creativecommons.org/licenses/ by-nc/4.0/), which permits any noncommercial use, distribution, and reproduction in any medium, provided you give appropriate credit to the original author(s) and the source, provide a link to the Creative Commons license, and indicate if changes were made.

\section{REFERENCES}

1. D'Amico A, Mercuri E, Tiziano FD, Bertini E. Spinal muscular atrophy. Orphanet J Rare Dis. 2011;6:71.

2. Ogino S, Leonard DGB, Rennert H, Wilson RB. Spinal muscular atrophy genetic testing experience at an academic medical center. J Mol Diagn. 2002;4(1):53-8.

3. Parente V, Corti S. Advances in spinal muscular atrophy therapeutics. Ther Adv Neurol Disord. 2018;11:1756285618754501.

4. Verhaart IEC, Robertson A, Wilson IJ, Aartsma-Rus A, Cameron S, Jones CC, et al. Prevalence, incidence and carrier frequency of 5q-linked spinal muscular atrophy-a literature review. Orphanet J Rare Dis. 2017;12(1):124.

5. Lefebvre S, Burglen L, Reboullet S, Clermont O, Burlet P, Viollet L, et al. Identification and characterization of a spinal muscular atrophy-determining gene. Cell. 1995;80(1):155-65.

6. Farrar MA, Kiernan MC. The genetics of spinal muscular atrophy: progress and challenges. Neurotherapeutics. 2015;12(2):290-302.

7. Monani UR. Spinal muscular atrophy: a deficiency in a ubiquitous protein; a motor neuron-specific disease. Neuron. 2005;48(6):885-96.

8. Faravelli I, Nizzardo M, Comi GP, Corti S. Spinal muscular atrophy-recent therapeutic advances for an old challenge. Nat Rev Neurol. 2015;11(6):351-9.

9. Iannaccone ST. Modern management of spinal muscular atrophy. J Child Neurol. 2007;22(8):974-8.

10. Taylor JE, Thomas NH, Lewis CM, Abbs SJ, Rodrigues NR, Davies KE, et al. Correlation of SMNt and SMNc gene copy number with age of onset and survival in spinal muscular atrophy. Eur J Hum Genet. 1998;6(5):467-74.

11. Darras BT. Spinal muscular atrophies. Pediatr Clin North Am. 2015;62(3):743-66.

12. Sproule DM, Hasnain R, Koenigsberger D, Montgomery M, De Vivo DC, Kaufmann P. Age at disease onset predicts likelihood and rapidity of growth failure among infants and young children with spinal muscular atrophy types 1 and 2. J Child Neurol. 2012;27(7):845-51.

13. Finkel RS, McDermott MP, Kaufmann P, Darras BT, Chung WK, Sproule DM, et al. Observational study of spinal muscular atrophy type I and implications for clinical trials. Neurology. 2014;83(9):810-7.

14. Glanzman AM, Mazzone E, Main M, Pelliccioni M, Wood J, Swoboda KJ, et al. The Children's Hospital of Philadelphia Infant Test of Neuromuscular Disorders (CHOP INTEND): test development and reliability. Neuromuscul Disord. 2010;20(3):155-61.

15. Bach JR. POINT: is noninvasive ventilation always the most appropriate manner of long-term ventilation for infants with spinal muscular atrophy type 1? Yes almost always. Chest. 2017;151(5):962-5.

16. Bach JR, Bakshiyev R, Hon A. Noninvasive respiratory management for patients with spinal cord injury and neuromuscular disease. Tanaffos. 2012;11(1):7-11. 
17. Kolb SJ, Coffey CS, Yankey JW, Krosschell K, Arnold WD, Rutkove SB, et al. Natural history of infantileonset spinal muscular atrophy. Ann Neurol. 2017;82(6):883-91.

18. US Food and Drug Administration. FDA approves first drug for spinal muscular atrophy. [Internet] Silver Spring, MD: FDA; 2016 [cited 2018 Mar 27]. http:// www.fda.gov/newsevents/newsroom/pressannounce ments/ucm534611.htm. Accessed 27 Mar 2018.

19. Finkel RS, Mercuri E, Darras BT, Connolly AM, Kuntz NL, Kirschner J, et al. Nusinersen versus sham control in infantile-onset spinal muscular atrophy. N Engl J Med. 2017;377(18):1723-32.

20. Luu KT, Norris DA, Gunawan R, Henry S, Geary R, Wang Y. Population pharmacokinetics of nusinersen in the cerebral spinal fluid and plasma of pediatric patients with spinal muscular atrophy following intrathecal administrations. J Clin Pharmacol. 2017;57(8):1031-41.

21. Mendell JR, Al-Zaidy S, Shell R, Arnold WD, RodinoKlapac LR, Prior TW, et al. Single-dose gene-replacement therapy for spinal muscular atrophy. N Engl J Med. 2017;377(18):1713-22.
22. Wijnhoven TM, de Onis M, Onyango AW, Wang T, Bjoerneboe GE, Bhandari N, et al. Assessment of gross motor development in the WHO multicentre growth reference study. Food Nutr Bull. 2004;25(1 Suppl):S37-45.

23. Talbot K, Tizzano EF. The clinical landscape for SMA in a new therapeutic era. Gene Ther. 2017;24(9):529-33.

24. Mercuri E, Finkel RS, Muntoni F, Wirth B, Montes J, Main M, et al. Diagnosis and management of spinal muscular atrophy: part 1: recommendations for diagnosis, rehabilitation, orthopedic and nutritional care. Neuromuscul Disord. 2018;28(2):103-15.

25. Wadman RI, Stam M, Gijzen M, Lemmink HH, Snoeck IN, Wijngaarde CA, et al. Association of motor milestones, SMN2 copy and outcome in spinal muscular atrophy types 0-4. J Neurol Neurosurg Psychiatry. 2017;88(4):365-7.

26. De Sanctis R, Pane M, Coratti G, Palermo C, Leone $\mathrm{D}$, Pera MC, et al. Clinical phenotypes and trajectories of disease progression in type 1 spinal muscular atrophy. Neuromuscul Disord. 2018;28(1): 24-8. 